\title{
Patterns of change in cytochrome c oxidase redox status
}

\author{
Roy E. Gagnon, Andrew J. Macnab* and Jacques G. LeBlanc \\ Department of Pediatrics, Department of Anesthesia, Department of Surgery, Children's \& Women's \\ Health Centre, University of British Columbia, Vancouver, BC, Canada
}

\begin{abstract}
Investigators using mono channel near infrared spectroscopy (NIRS) have reported different patterns of change in cytochrome c oxidase (Cyt) in similar studies of tissue ischaemia. We investigated whether there were distinctive differences in NIRS signals obtained simultaneously from different sampling sites during the same imposed physiological intervention within the same subject. Methods: Subjects were 36, healthy, $10 \mathrm{~kg}$, commercial swine undergoing cardiopulmonary bypass to initiate 3 to 7 periods of 7.5 minutes of circulatory arrest. Each arrest was initiated at one of 81 combinations of high, normal, or low levels of core temperature, haematocrit, $\mathrm{pH}$, and serum glucose. Each combination was repeated twice, yielding 162 NIRS data sets. Results: Six distinct patterns of change of Cyt were found. Typically, brain Cyt quickly became reduced shortly after the start of arrest, muscle Cyt did not start becoming reduced until after $3 \frac{1}{2}$ minutes of arrest, and spinal cord Cyt either did not change status or became gradually reduced throughout the period of arrest. The brain response may reflect strong oxygen dependence, while the muscle response may indicate a dependency buffered by myoglobin stores, and the spine response may indicate a low concentration of available Cyt that is too diffuse to be rapidly influenced by changes in oxygen availability. Conclusion: Multi-channel NIRS is needed for systemic evaluation of respiration at the cellular level in clinical settings. Distinctive Cyt patterns of change occur in different organs at the same time, in response to circulatory arrest.
\end{abstract}

\section{Introduction}

This study examines whether changes in electron transfer within Complex IV of the mitochondrial respiratory chain in response to total circulatory arrest differs in the spine, brain and muscle as measured by near infrared spectrophotometry.

Noninvasive near infrared spectrophotometry (NIRS) has now been available for experimentation in animals and humans for several decades. To date, NIRS has been used primarily to measure patterns of change in the concentrations of oxygenated hemoglobin $\left(\mathrm{HbO}_{2}\right)$ and de-oxygenated hemoglobin $(\mathrm{Hb})$ in the blood, and to measure the redox status of cytochrome a, $\mathrm{a}_{3}(\mathrm{Cyt})$. Cytochrome $\mathrm{a}, \mathrm{a}_{3}$, residing on the cristae of the intracellular mitochondria, is the terminal enzyme in the respiratory chain and, as a catalyst promoting the release of energy, its redox status reflects mitochondria oxygen supply and demand [7].

There have been a number of trials validating the NIRS cytochrome $\mathrm{a}_{3} \mathrm{a}_{3}$ signal, but results have sometimes varied between investigators $[1-3,5,9,11,13]$. For example, it was found that Cyt redox status corresponds directly with changes in $\mathrm{HbO}_{2}$ in human muscle, but the opposite was found in horse muscle $[4,10]$.

\footnotetext{
*Corresponding author: Prof. A.J. Macnab, Director, Critical Care Research Office, Room L317, Mail Box 80, Children's \& Women's Health Centre of BC, 4480 Oak St., Vancouver, BC, Canada, V6H 3N1. E-mail: amacnab@cw.bc.ca.
} 
In this study, we used NIRS to investigate whether there were distinctive differences in the Cyt redox patterns of change obtained simultaneously from diverse sampling sites during the same imposed physiological intervention within the same subject.

\section{Method}

The subjects were 36 healthy $10 \mathrm{~kg}$ juvenile Yorkshire swine. The experimental protocol was approved by the University of British Columbia's Animal Care Committee. All animals were anesthetized, intubated, mechanically ventilated, and placed on mechanical cardiopulmonary bypass.

All animals had the sensor array of a NIRO-300 (Hamamatsu Photonics KK, Hamamatsu City, Japan) spectrophotometer positioned on the intact skin of the skull at the intersection of the left-eye/left-ear lateral and longitudinal axes. It was oriented towards the NIRO-300's emitter which was placed similarly at the right-eye/right-ear axis intersection $55 \mathrm{~mm}$ from the sensor. The second channel of the NIRO-300 was placed with its sensor on the intact skin over the mid thoracic spine and its emitter on the intact skin over the spine $65 \mathrm{~mm}$ caudal. The emitter and detector optodes of a NIRO-500 (Hamamatsu Photonics KK, Hamamatsu City, Japan) spectrophotometer were placed on the intact skin along the midline of the right hind flank with a $40 \mathrm{~mm}$ inter-optode spacing. Once in place, the sensor/emitters on the brain, spine, and muscle were covered with light occluding masks. Both the NIRO-500 and NIRO-300 were set to collect data continuously at $1 \mathrm{~Hz}$.

During bypass, each animal had two or more $71 / 2$ minute periods of circulatory arrest. Before each period of arrest, the animal's body temperature, haematocrit, $\mathrm{pH}$, and glucose were adjusted to match a randomly chosen combination of ranges. Body temperature was established at 22,18 , or $14^{\circ} \mathrm{C}$, haematocrit at 14,20 , or $26 \%, \mathrm{pH}$ at $7.25,7.40$ or 7.55 , and glucose was established at $4.7,6.0$ or $7.25 \mathrm{mmol} / \mathrm{l}$. There were 81 combinations of physiologic parameters that were repeated twice among all 36 animals.

Blood samples were taken to measure sodium, potassium, bicarbonate, glucose, $\mathrm{pH}$, haematocrit, partial pressure of carbon dioxide, partial pressure of oxygen, base excess, and oxygen saturation. These were recorded by iSTAT (Abbott Laboratories, Abbott Park, Illinois USA) point-of-care analyzers within three minutes of withdrawal.

Data were analyzed using Excel-XP (Microsoft Canada Co. Mississauga, Ontario), and SPSS 11.01 (SPSS Inc., Chicago IL) was used for the 4-way ANOVA testing.

\section{Results}

There were $11(7 \%)$ spine and muscle graphs that were incomplete because of blood and fluid seepage contaminating the sensor(s) or because the skin adhesive failed and the sensor(s) became displaced. These partial data sets were excluded from the analysis.

$\mathrm{The} \mathrm{HbO}_{2}$ and $\mathrm{Hb}$ patterns of change were the same for all trials and had the same pattern of change in the brain as in the spinal cord and muscle. During the period of arrest, $\mathrm{HbO}_{2}$ concentration decreased while $\mathrm{Hb}$ concentration increased.

The Cyt patterns of change in the brain, spine, and muscle could be categorized into 6 distinctive patterns as shown in Fig. 1. The distribution of the Cyt patterns of change obtained given by the percentage of classification category is shown in Fig. 2.

There was no period of circulatory arrest in which the brain, spine, and muscle all had the same Classification Type of Cyt pattern of change. In approximately 35\% of muscle data sets, $15 \%$ of cerebral 


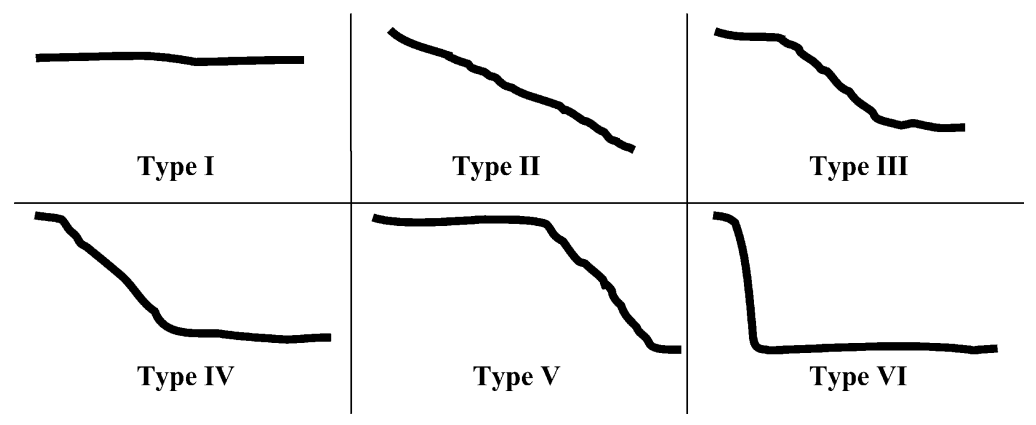

Fig. 1. Types of cytochrome a, $a_{3}$ patterns of change during $71 / 2$ minutes of circulatory arrest. The rates of change for the slopes shown are given in Table 1.

Table 1

Summary of the rates of change for the types of cytochrome $\mathrm{a}_{3} \mathrm{a}_{3}$ patterns of change shown in Fig. 1

\begin{tabular}{|c|c|c|c|c|}
\hline Tissue & Pattern of change type & Number of arrests & $\begin{array}{l}\text { Rate of change average } \\
\left(\mu \mathrm{mol} \cdot 1^{-1} \cdot \min ^{-1}\right)\end{array}$ & $\begin{array}{c}\text { Rate of change standard deviation } \\
\left(\mu \mathrm{mol} \cdot 1^{-1} \cdot \min ^{-1}\right)\end{array}$ \\
\hline$\overline{\text { Brai }}$ & I & 11 & 0.00 & \pm 0.00 \\
\hline Brain & II & 10 & -0.18 & \pm 0.06 \\
\hline Brain & III & 22 & -0.36 & \pm 0.12 \\
\hline Brain & IV & 13 & -0.24 & \pm 0.12 \\
\hline Brain & V & 25 & -0.36 & \pm 0.12 \\
\hline \multirow[t]{2}{*}{ Brain } & VI & 81 & -0.72 & \pm 0.42 \\
\hline & Total & 162 & & \\
\hline Muscle & I & 19 & 0.00 & \pm 0.00 \\
\hline Muscle & II & 32 & -0.24 & \pm 0.12 \\
\hline Muscle & III & 11 & -0.42 & \pm 0.18 \\
\hline Muscle & IV & 1 & -0.36 & - \\
\hline Muscle & V & 63 & -0.48 & \pm 0.24 \\
\hline \multirow[t]{2}{*}{ Muscle } & VI & 25 & -1.14 & \pm 0.42 \\
\hline & Total & 151 & & \\
\hline Spine & I & 91 & 0.00 & \pm 0.00 \\
\hline Spine & II & 46 & -0.18 & \pm 0.06 \\
\hline Spine & III & 5 & -0.24 & \pm 0.06 \\
\hline Spine & IV & 4 & -0.26 & \pm 0.06 \\
\hline Spine & V & 0 & - & - \\
\hline \multirow[t]{2}{*}{ Spine } & VI & 5 & -0.96 & \pm 0.36 \\
\hline & Total & 151 & & \\
\hline
\end{tabular}

data sets, and $75 \%$ of spine data sets, Cyt redox status either did not change or became gradually reduced. Most (44\% of 162) muscle data sets did not show reduction until after the first $31 / 2$ minutes of arrest, while most (49\% of 162) brain collections showed reduction soon after the start of arrest.

In the majority $(60 \%)$ of spine collections, Cyt changes (Type I) were markedly different from the majority (50\%) in the brain (Type VI), and the majority (43\%) in muscle (Type V), with a rate of change in the spine at least 100 times slower than either the brain or muscle. 


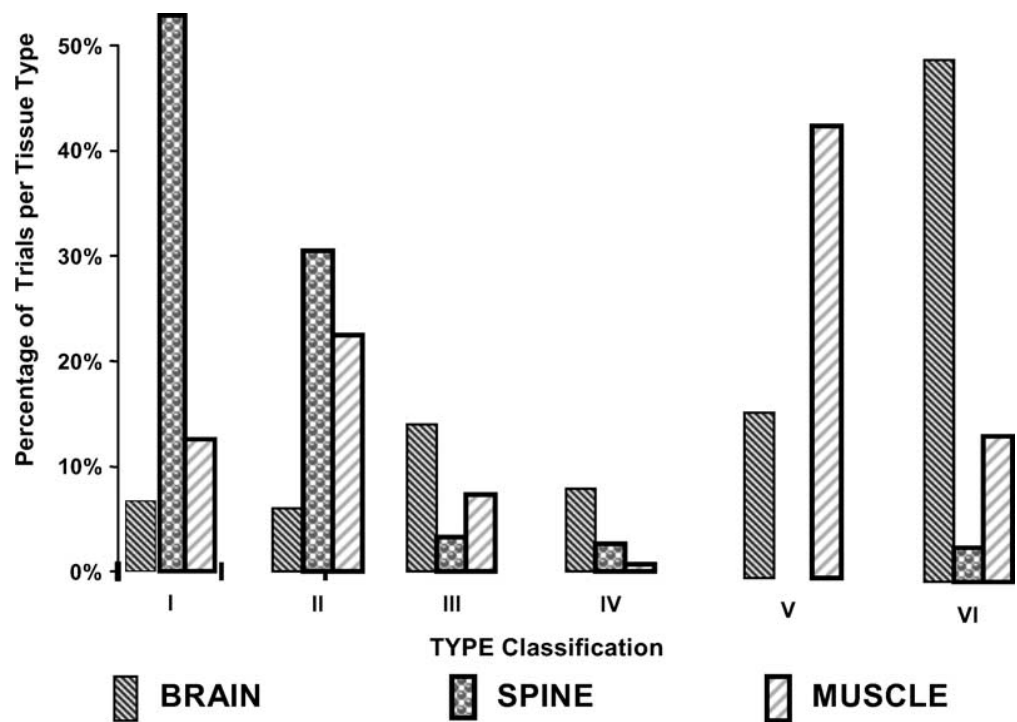

Fig. 2. Histogram of the occurrence of types of patterns of change given in Fig. 1 from 162 brain, 131 brain, and 151 muscle trials among 36 healthy anesthetized swine.

\section{Discussion}

This study used 3 NIRS channels to simultaneously monitor brain, spine, and muscle tissue oxygenation status and found that the patterns of change in Cyt redox always differ between organs.

Conventionally, it is assumed that all Cyt enzymes are structurally alike whether isolated from wheatgrass root, bovine heart, or bacteria; and by corollary, behave alike. While their spectra have been found to be alike in the neonatal pig, adult rat, and purified enzyme, it remains possible that significant spectral differences still exist $[2,8]$. Investigators do not yet agree on the electron exchange rates, or the conformation of the molecule, or its electron transfer pathways and proton interactions [12,14]. Thus a static model of Cyt is not yet complete, while a behavioral model is only in its early stages of understanding [6]. The behavior of this enzyme in isolation may be entirely different from that of an extensive Cyt in vivo cohort. Given these many uncertainties in understanding Cyt, it is possible that while Cyt enzymes may be alike between species, their patterns of change may be unalike given different tissue environments.

The four circulatory arrest parameters (temperature, haematocrit, $\mathrm{pH}$, and glucose) were established and measured in circulating blood. However, interstitial $\mathrm{pH}$ and glucose were not measured and may have differed from the blood. The 4-way analysis of variance (ANOVA) results shown in Table 2 indicate that only temperature is related to the rate of change in Cyt regardless of sampling site. This more likely indicates that additional trials are needed in order to increase the power of detection within this test, rather than that any cross relationship exclusively does not exist.

The finding that 6 Cyt patterns of change are common to each organ and that only 6 patterns were found in 162 trials, suggests a potential usefulness in developing artificial intelligence software for automated interpretation of NIRS results. A graphical index of typical patterns of change versus species, versus organ, and versus intervention, could be created to enhance the interpretation of clinical NIRS observations. 
Table 2

Summary of the 4-way ANOVA testing for the rates of change as a dependent variable with temperature, haematocrit, $\mathrm{pH}$, and serum glucose as independent variables

\begin{tabular}{lccc}
\hline Rate-of-change factors & \multicolumn{3}{c}{$P$-value $(\alpha=0.050)$} \\
\cline { 2 - 4 } & Brain & Spine & Muscle \\
\hline Temperature (Temp) & 0.003 & 0.056 & 0.445 \\
Haematocrit (Hct) & 0.135 & 0.724 & 0.204 \\
pH (pH) & 0.159 & 0.738 & 0.126 \\
Glucose (Glu) & 0.471 & 0.814 & 0.386 \\
Temp \& Hct & 0.532 & 0.189 & 0.182 \\
Temp \& pH & 0.143 & 0.884 & 0.404 \\
Temp \& Gluc & 0.806 & 0.431 & 0.835 \\
Hct \& pH & 0.519 & 0.152 & 0.178 \\
Hct \& Gluc & 0.910 & 0.336 & 0.103 \\
pH \& Gluc & 0.964 & 0.168 & 0.315 \\
Temp \& Hct \& pH & 0.510 & 0.371 & 0.648 \\
Temp \& Hct \& Gluc & 0.803 & 0.440 & 0.137 \\
Temp \& pH \& Gluc & 0.994 & 0.560 & 0.988 \\
Hct \& pH \& Gluc & 0.977 & 0.324 & 0.842 \\
Temp \& Hct \& pH \& Gluc & 0.992 & 0.929 & 0.633 \\
\hline
\end{tabular}

\section{Conclusion}

Multi-channel NIRS is needed for systemic in vivo evaluation of respiration at the cellular level. The respiratory chromophore, cytochrome c oxidase, has nine typical patterns of change in redox status none of which appears simultaneously in the brain, spinal cord, and/or muscle as a response to circulatory arrest.

\section{Acknowledgements}

We wish to thank the British Columbia \& Yukon Heart and Stroke Foundation for their financial support.

\section{References}

[1] C.E. Cooper and R. Springett, Measurement of cytochrome oxidase and mitochondrial energetics by near-infrared spectroscopy, Philos. Trans. R. Soc. Lond. B Biol. Sci. 352(1354) (1997), 669-676.

[2] C.E. Cooper, D.T. Delpy and E.M. Nemoto, The relationship of oxygen delivery to absolute haemoglobin oxygenation and mitochondrial cytochrome oxidase redox state in the adult brain: a near-infrared spectroscopy study, Biochem. J. 332(pt3) (1998), 627-632.

[3] C.E. Cooper, M. Cope, R. Sprigett, P.N. Amess, J. Penrice, L. Tyszczuk, S. Punwani, R. Ordidge, J. Wyatt and D.T. Delpy, Use of mitochondrial inhibitors to demonstrate that cytochrome oxidase near-infrared spectroscopy can measure mitochondrial dysfunction noninvasively in the brain, J. Cereb. Blood Flow Metab. 19(1) (1999), 27-38.

[4] J. Hansen, G.D. Thomas, S.A. Harris, W.J. Parsons and R.G. Victor, Differential sympathetic neural control of oxygenation in resting and exercising human skeletal muscle, J. Clin. Invest. 98 (1996), 584-596.

[5] H.R. Heekeren, M. Kohl, H. Obrig, R. Wenzel, W. von Pannwitz, S.J. Matcher, U. Dirnagl, C.E. Cooper and A. Villringer, Noninvasive assessment of changes in cytochrome-c oxidase oxidation in human subjects during visual stimulation, J. Cereb. Blood Flow Metab. 19(6) (1999), 592-603. 
[6] B. Korzeniewski, Thermodynamic regulation of cytochrome oxidase, Mol. Cell. Biochem. 174 (1997), 137-141.

[7] S.M. Musser and S.I. Chan, Evolution of the cytochrome c oxidase proton pump, J. Mol. Evol. 46(5) (1998), 508-520.

[8] P. Nicholls, The extinction coefficients for cytochrome $a_{3}$ and their consequences, 2001; http://www-bioc.rice.edu/ $\sim$ graham/Nicholls.pdf.

[9] G. Nollert, R.A. Jonas and B. Reichart, Optimizing cerebral oxygenation during cardiac surgery: a review of experimental and clinical investigations with near infrared spectroscopy, Thorac. Cardiovasc. Surg. 48(4) (2000), 247-253.

[10] J. Pringle, C. Roberts, T. Art and P. Lekeux, Assessment of muscle oxygenation in the horse by near infrared spectroscopy, Equine Vet. J. 32(1) (2000), 59-64.

[11] V. Quaresima, R. Springett, M. Cope, J.T. Wyatt, D.T. Delpy, M. Ferrari and C.E. Cooper, Oxidation and reduction of cytochrome oxidase in the neonatal brain observed by in vivo near-infrared spectroscopy, Biochim. Biophys. Acta 1366(3) (1998), 291-300.

[12] M. Saraste, Oxidative phosphorylation at the fin de siècle, Science 283 (1999), 1488-1493.

[13] M. Shadid, L. Hiltermann, L. Monteiro, J. Fontijn and F. Van Bel, Near infrared spectroscopy—measured changes in cerebral blood volume and cytochrome $\mathrm{aa}_{3}$ in newborn lambs exposed to hypoxia and hypercapnia, and ischemia: a comparison with changes in brain perfusion and $\mathrm{O}_{2}$ metabolism, Early Hum. Dev. 55(2) (1999), 169-182.

[14] T. Tsukihara, H. Aoyama, E. Yamashita, T. Tomizaki, H. Yamaguchi, K. Shinzawa-itoh, R. Nakashima, R. Yaono and S. Yoshikawa, Structures of metal sites of oxidized bovine heart cytochrome c oxidase at $2.8 \AA$, Science 269 (1995), 1069-1074. 


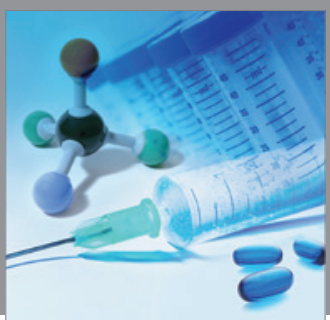

International Journal of

Medicinal Chemistry

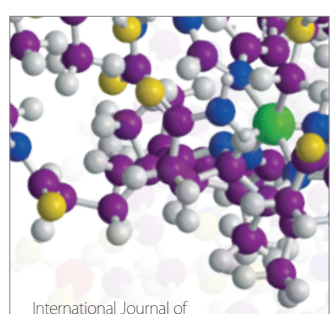

Carbohydrate Chemistry

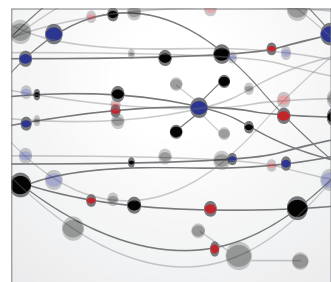

The Scientific World Journal
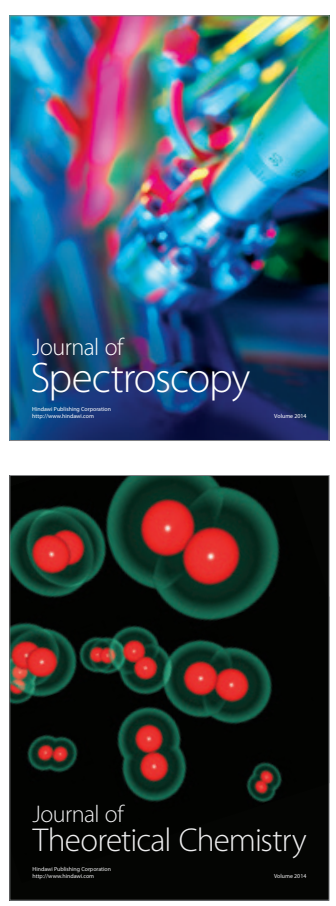
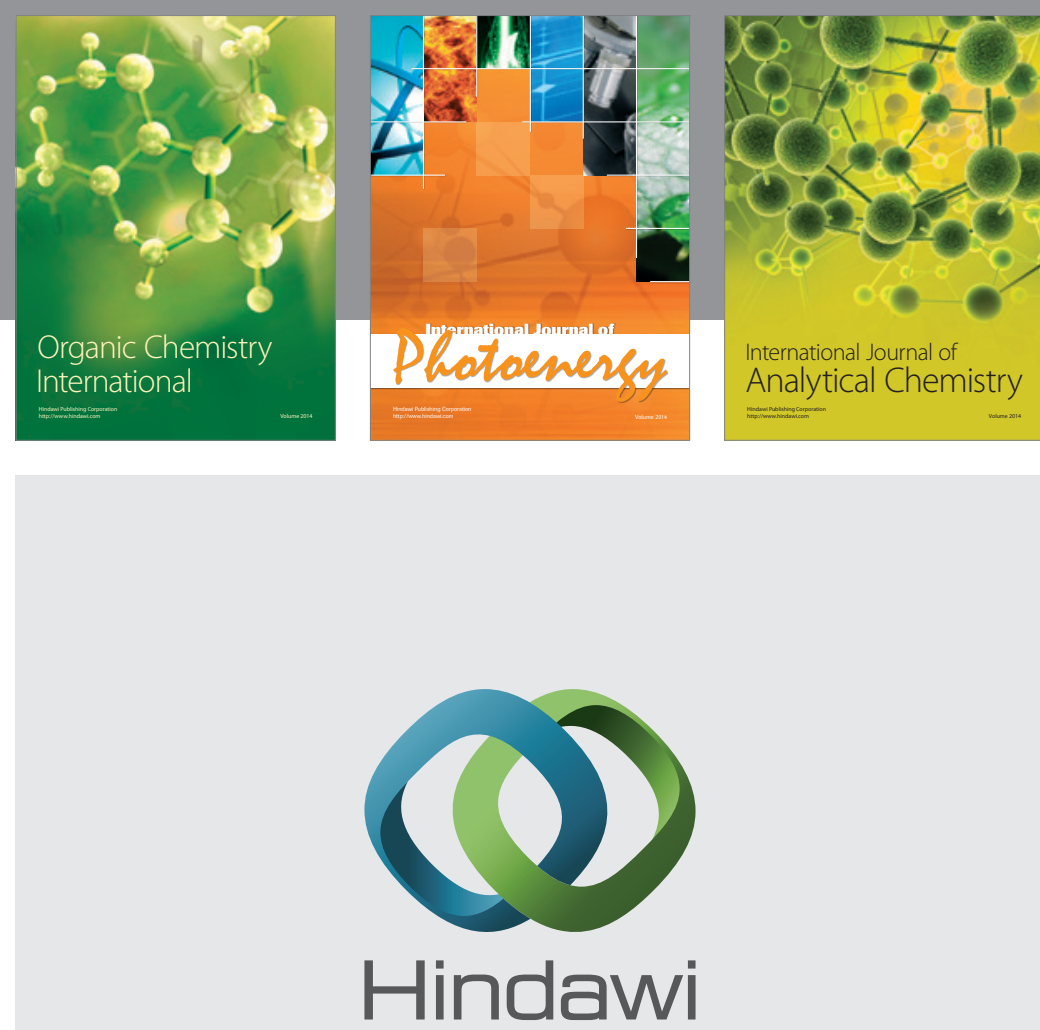

Submit your manuscripts at

http://www.hindawi.com
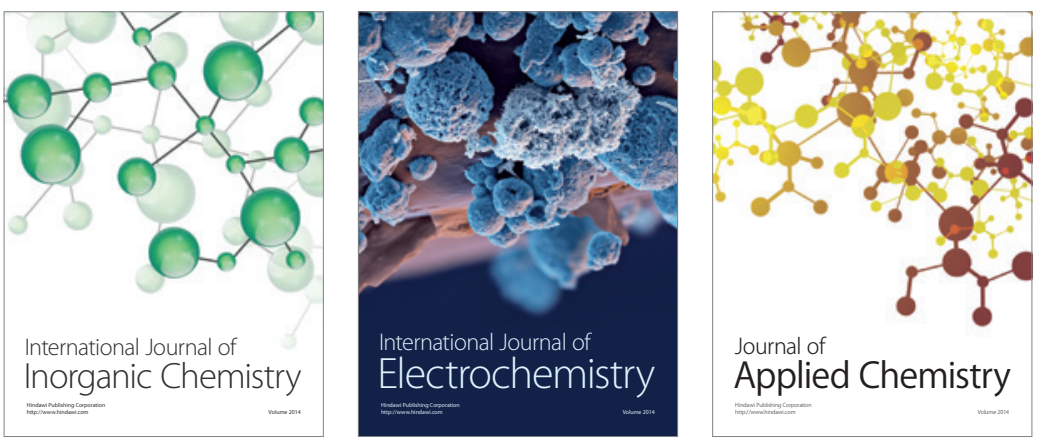

Journal of

Applied Chemistry
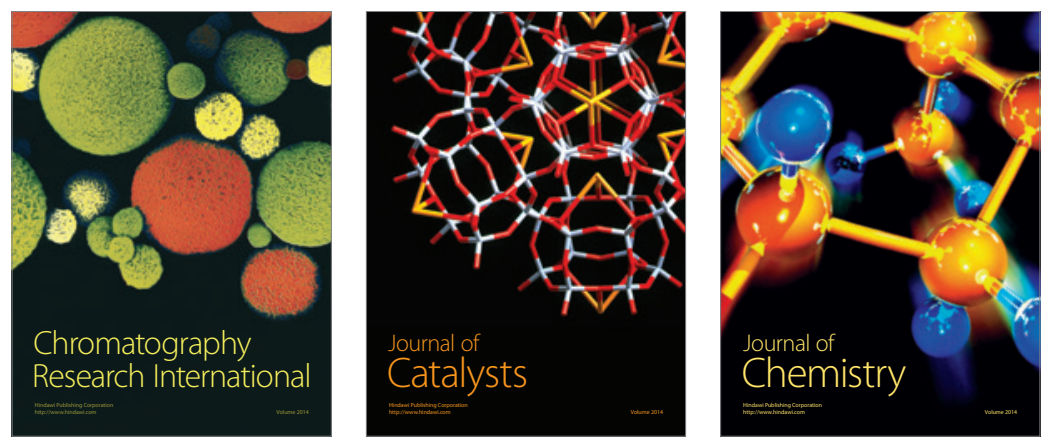
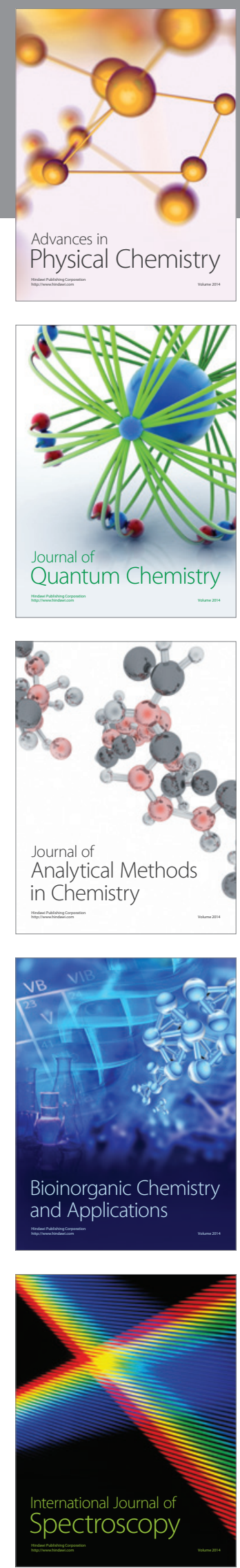